\title{
New Equipment for Correlative FIB/TEM/Atom Probe and Site-Specific Preparation Using STEM Live Imaging.
}

\author{
P. Felfer and J. M. Cairney
}

Australian Centre for Microscopy and Microanalysis, The University of Sydney, NSW 2006, Camperdown, Australia.

In many atom probe projects, site-specific sample preparation is the key to obtaining the desired information. These days, focused ion beam (FIB)-scanning electron microscopy (SEM) platforms are usually used for this purpose. Even though many FIB-SEMs are equipped with field-emission SEM columns, the absence of contrast from a targeted feature such as a grain boundary can render the task of site-specific preparation difficult. Analysis of tips with the TEM can help to improve the situation. Also, cross-correlative studies with TEM can yield valuable information needed for correct interpretation of the atom probe results. However, the shape of atom probe samples makes them prone to damage at any point of handling.

We have therefore designed clamp holders that fit both wire-shaped and disk-shaped samples and can be used in FIB, TEM and Cameca LEAP ${ }^{\circledR}$ atom probe platforms, drastically reducing the risk of damage (fig. 1). In order to be able to attach multiple specimens from a lift-out bar onto a support, electropolished Mo-TEM grids are used. The holders are adapted to so-called 'dummy holders' that are commonly used with JEOL TEMs, but could easily be adapted to TEMs from other manufacturers. Similar commercial systems are also available [1]. These holders can be used both to produce site-specific samples in FIB-SEM systems in which the resolution of the e-beam doesn't allow for the clear imaging of AP tips and for correlative studies in dedicated TEMs.

For the task of site-specific preparation, the use of a dedicated TEM is not always required. Nevertheless, the contrast produced by transmitted electrons (STEM) in is very often better suited than secondary electrons to image the region of interest within an atom probe sample (fig. 2 is showing a grain boundary in a steel). They allow a look at the inside of the specimen, revealing the precise position of buried features such as micro-devices. In a FIB-SEM (Zeiss Auriga ${ }^{\mathbb{B}}$ ) system, equipped with a FE-SEM column, a retractable STEM detector has been installed in conjunction with an additional rotational axis with a freestanding custom-designed specimen cartridge at the end that can hold wire-shaped and disk-shaped specimens and be transferred to the atom probe. The STEM detector fits underneath the specimen cartridge, which can rotate by a full $360^{\circ}$. This way, all manipulation steps of the lift-out procedure can be carried out without breaking the vacuum. Using transmitted electrons, the milling progress can be monitored live without the problem of noise being created by secondary electrons stemming from the ion beam. The milling can then be stopped precisely when the apex of the atom probe specimen is at the desired distance from the region of interest.

References

[1] B. Gorman et al., Microscopy today. Vol. 16/4 (2008) 42.

[2] The Authors would like to thank H. Felfer for help during the manufacturing of the prototypes of both systems. 


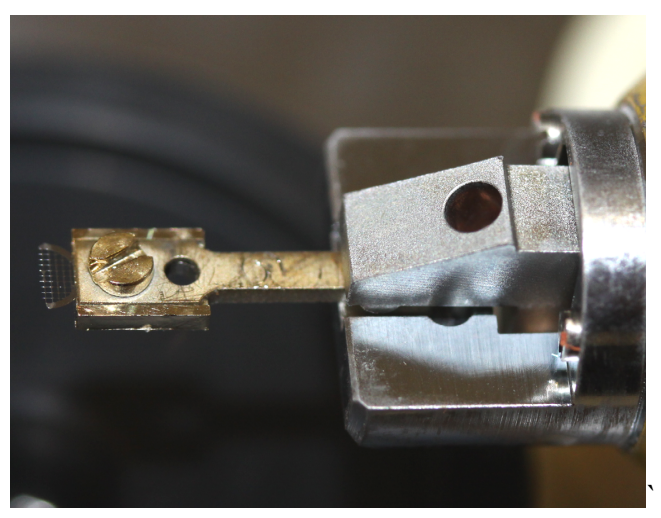

FIG. 1. Specimen cartridge compatible to FIB/TEM/LEAP, attached to a JEOL 'dummy holder'

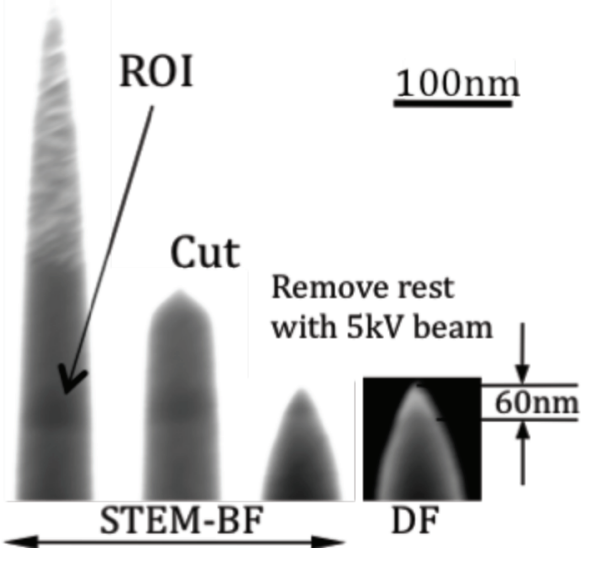

FIG. 2. Positioning of a grain boundary in a steel for atom probe analysis using a STEM detector inside a FIB-SEM. 